Original Article

\title{
Investigation of the effects of mirror therapy on the upper extremity functions of stroke patients using the manual function test
}

\author{
Hwanhee Kim, PhD, $\mathrm{OT}^{1)}$, Jemyung Shim, PhD, $\mathrm{PT}^{2)^{*}}$ \\ 1) Department of Occupational Therapy, Semyung University, Republic of Korea \\ 2) Department of Physical Therapy, College of Health and Science, Kangwon National University: 346 \\ Hwangjo-ri, Dogye-eup Samcheok-si, Gangwon-do 245-905, Republic of Korea
}

\begin{abstract}
Purpose] The purpose of this study was to investigate the effects of mirror therapy on the upper extremity functions of stroke patients. [Subjects] The subjects of this study were 14 hemiplegia patients ( 8 males, 6 females; 9 infarction, 5 hemorrhage; 8 right hemiplegia, 6 left hemiplegia) who voluntarily consented to participate in the study. [Methods] The Korean version of the manual function test (MFT) was used in this study. The test was performed in the following order: arm movement (4 items), grasp and pinch ( 2 items), and manipulation (2 items). The experiment was conducted with the subjects sitting in a chair. The mirror was vertically placed in the sagittal plane on the desk. The paretic hand was placed behind the mirror, and the non-paretic hand was placed in front of the mirror so that it was reflected in the mirror. In this position, the subjects completed activities repetitively according to the mirror therapy program over the course of four weeks. [Results] There were significant increases in the grasp-and-pinch score and manipulation score. [Conclusion] In conclusion, the grasp-and-pinch and manipulation functions were improved through mirror therapy.

Key words: Mirror therapy, Manual function test, Stroke
\end{abstract}

(This article was submitted Jun. 25, 2014, and was accepted Aug. 3, 2014)

\section{INTRODUCTION}

Among the diverse neurological disorders that appear after a stroke, upper extremity disabilities are one of the most common. These disabilities can include decreased motor ability and restriction in the functional use of the paretic upper extremity, resulting in weakening or paralysis of the muscles, abnormal muscle tone, associated reaction, problems with the musculoskeletal system, and coordination disorder ${ }^{1}$. Dysfunctions from upper extremity hemiparesis impair the performance of many daily activities such as dressing, bathing, self-care, and writing, thus reducing functional independence ${ }^{2}$. The functions of the upper extremities and hands are some of the most important functions for performing daily living and working activities.

Since 2000, mirror therapy has been much applied and studied as a method to treat the upper extremities of stroke patients. Mirror therapy in stroke patients involves performing movements of the unimpaired limb while watching its mirror reflection superimposed over the (unseen) impaired limb, thus creating a visual illusion of enhanced movement capability in the impaired limb $b^{3}$. This is a therapeutic inter-

*Corresponding author. Jemyung Shim (E-mail: sjm7897@, hanmail.net)

(C2015 The Society of Physical Therapy Science. Published by IPEC Inc. This is an open-access article distributed under the terms of the Creative Commons Attribution Non-Commercial No Derivatives (by-ncnd) License $<$ http://creativecommons.org/licenses/by-nc-nd/3.0/>. vention that uses visual feedbacks for neuroplasticity and triggers motivation through visual feedbacks during training ${ }^{4,5)}$.

Voluntary movement of the paretic upper extremity and hand using a mirror activates the bilateral cortex and causes reorganization in other areas around the damaged part of the brain, thus allowing for replacement of its function and thereby affecting motor function recovery ${ }^{6}$. Therefore, it is accepted as an epoch-making method in the clinical field whereby a therapist provides an intervention and manual treatment in a person-to-person manner.

Therefore, this study examined the degree to which stroke patients can regain upper-extremity functioning using mirror therapy.

\section{SUBJECTS AND METHODS}

The subjects of this study were 14 hemiplegia patients ( 8 males, 6 females; 9 infarction, 5 hemorrhage; 8 right hemiplegia, 6 left hemiplegia) who voluntarily consented to participate in the study. Their average age was $56.43 \pm$ 10.68 years, their average MMSE-K was $29.71 \pm 0.47$, and their average poststroke duration was $7.29 \pm 1.59$ months. This study was approved by the Ethics Review Committee of Kangwon National University (approval number KWNUH 2013-09-003). The study was performed from September 1, 2012, to October 10, 2013. The location of the experiment was an occupational therapy room in the rehabilitation center of $\mathrm{D}$ Clinic in Busan, Republic of 
Korea. The inclusion criteria for the stroke patients were as follows: 1) those who were diagnosed by a doctor with hemiplegia resulting from a stroke; 2) those who did not undergo brain surgery; 3) those with a Mini-Mental Status Examination-Korea (MMSE-K) score of 24 or higher who were proven to have no cognitive function disorder; 4) those who were proven to have no disabilities in visual perceptive functions including neglect by the Motor-Free Visual Perceptual Test-Revised; and 5) those whose stroke occurred at least 6 months prior to the study.

The Korean version of the manual function test (MFT), translated by $\mathrm{Kim}^{7)}$, was used in the present study. The time needed for evaluation in this test is short (about 10 minutes), and it can be applied easily. The MFT makes used of goniometers, a ball, a coin, a needle, a pencil, a board for carrying board cubes, a pegboard and pegs, a stopwatch, and a sheet. The test is performed in the following order: arm movement (4 items), grasp and pinch ( 2 items), and manipulation (2 items). The MFT was employed as an evaluation tool prior to and after the intervention. Two testers conducted the evaluation. The paretic side was assessed after the non-paretic side was evaluated. The evaluation method was explained to the subjects so that they fully understood it.

The mirror therapy program was reorganized as an intervention by the researcher to be suitable for the purpose of this study and was based on a program by Yavuzer et al. ${ }^{8)}$ and St. Gallen Protocol for mirror therapy ${ }^{9}$. The experiment was conducted while the subjects sat in a chair. The mirror was placed vertically in the sagittal plane on the desk. The paretic hand was placed behind the mirror, and the non-paretic hand was placed in front of the mirror so that it was reflected in the mirror. When the program began, the patients observed the upper extremity of the non-paretic side reflected in the mirror. In this position, they conducted the activities repetitively according to the mirror therapy program. The patients conducted activities by themselves, but under the supervision of a therapist. After four weeks, the MFT score for each subject was measured using the same processes as used prior to the intervention.

For the data analysis in this study, SPSS 20.0 version was used for the statistical processing. In the upper extremity function test, a paired t-test was used to analyze differences between prior to mirror therapy and four weeks after the therapy. The significance level was set at $\alpha=0.05$.

\section{RESULTS}

The scores in all areas of the MFT increased after the intervention compared with before the intervention. The grasp-and-pinch score was $2.50 \pm 2.07$, thus showing a statistically significant difference $(\mathrm{p}<0.05)$. The arm-movement score was $9.00 \pm 4.28$ prior to the intervention and increased to $9.64 \pm 4.83$ after the intervention, which was not a statistically significant difference $(p>0.05)$. The score for all the activities was $13.15 \pm 7.55$ prior to the intervention and $16.71 \pm 7.55$ after the intervention, which is not a statistically significant difference $(\mathrm{p}>0.05)$ (Table 1).
Table 1. Comparison of upper extremity function before and after mirror therapy in the patients (Unit: score)

\begin{tabular}{lccc}
\hline \multirow{2}{*}{ MFT } & Before & After \\
\cline { 2 - 3 } & Mean \pm SD & Mean \pm SD & \\
\hline Arm movement & $9.00 \pm 4.28$ & $9.64 \pm 4.83$ & \\
Grasp and pinch & $2.50 \pm 2.07$ & $4.00 \pm 2.00$ & $*$ \\
Manipulation & $1.64 \pm 1.65$ & $2.79 \pm 1.53$ & $*$ \\
Total & $13.15 \pm 7.55$ & $16.71 \pm 7.55$ & \\
\hline
\end{tabular}

\section{DISCUSSION}

After a stroke, 55 to $75 \%$ of stroke patients have a paretic arm that causes motor impairments ${ }^{10)}$ and experience difficulty in incorporating the affected hand into their daily activities $^{11)}$. In order to improve such upper extremity functions, researchers have been experimenting with mirror therapy. This study employed the MFT to examine the effects of mirror therapy on upper extremity functions.

The present study showed that the scores for all of the MFT activities increased after the intervention using mirror therapy. Among them, there were statistically significant differences in the grasp-and-pinch and manipulation scores $(p<0.05)$, but there were no statistically significant differences in the arm-movement and total scores $(\mathrm{p}>0.05)$. Therefore, the grasp-and-pinch and manipulation functions were improved through mirror therapy. However, the size of the mirror $(35 \times 35 \mathrm{~cm})$ is considered too small to observe the range of motion of the upper extremity; therefore, upper extremity movements may differ according to the position and functions of the trunk, making it difficult to the enhance the range of motion of the upper extremity with mirror therapy alone. As a result, the sum of these three items may have been affected.

A previous study that applied mirror therapy to chronic stroke patients for 20 sessions also found that there was only improvement in the arm-movement (raising the upper extremity forward) and grasp-and-pinch (grasping, moving a cube) functions ${ }^{12)}$. However, when the upper extremity function test was conducted after mirror therapy using the MFT with subacute stroke patients as the subjects, there were significant improvements in all items ${ }^{13-15)}$.

This study has some limitations. First, the stroke patients were hospitalized, making it hard to control the environment outside the treatment room. Second, the mirror therapy program was conducted independently, meaning some subjects were bored due to spending the 30-minute observation time alone. Prior research has noted that mirror therapy demands high levels of concentration and may be boring; therefore, it is difficult to expect active participation ${ }^{9}$. In this study, the supervising therapist motivated the subjects from time to time.

Future research should attempt to recify the limitations mentioned above. This study only examined chronic stroke patients; therefore, a higher number of patients with diverse diseases and conditions, including sensory damages in the upper extremity and burns, should be included in future studies. 


\section{REFERENCES}

1) Woodson AM: Stroke. In: Radomski MV, Trombly Latham CA (Eds.), Occupational Therapy for Physical Dysfunction, 6th ed. Philadelphia: Lippincott Williams \& Wilkins, 2008, pp 1001-1041.

2) Gillen G: Cerebrovascular accident/stroke. In: Pendleton HM \& SchultzKrohn W (Eds.), Pedretti's occupational therapy: Practice skills for physical dysfunction, 7th ed. Philadelphia: Mosby, 2012, pp 844-880.

3) Stevens JA, Stoykov ME: Using motor imagery in the rehabilitation of hemiparesis. Arch Phys Med Rehabil, 2003, 84: 1090-1092. [Medline] [CrossRef]

4) Jack D, Boian R, Merians AS, et al.: Virtual reality-enhanced stroke rehabilitation. IEEE Trans Neural Syst Rehabil Eng, 2001, 9: 308-318. [Medline] [CrossRef]

5) Ji SG, Cha HG, Kim MK, et al.: The effect of mirror therapy integrating functional electrical stimulation on the gait of stroke patients. J Phys Ther Sci, 2014, 26: 497-499. [Medline] [CrossRef]

6) Thirumala P, Hier DB, Patel P: Motor recovery after stroke: lessons from functional brain imaging. Neurol Res, 2002, 24: 453-458. [Medline] [CrossRef]

7) Kim MY: A study of manual functional test for CVA. J Korean Soc Occup Ther, 1994, 26: 19-26.

8) Yavuzer G, Selles R, Sezer N, et al.: Mirror therapy improves hand function in subacute stroke: a randomized controlled trial. Arch Phys Med Re- habil, 2008, 89: 393-398. [Medline] [CrossRef]

9) Grunert-Pluss N, Hufschmid U, Santschi L, et al.: Mirror therapy in hand rehabilitation: a review of the literature, the St Gallen protocol for mirror therapy and evaluation of a case series of 52 patients. Br J Hand Ther, 2008, 13: 4-11.

10) Jørgensen HS, Nakayama H, Raaschou HO, et al.: Outcome and time course of recovery in stroke. Part II: Time course of recovery. The Copenhagen stroke study. Arch Phys Med Rehabil, 1995, 76: 406-412. [Medline] [CrossRef]

11) Dobkin BH: Clinical practice. Rehabilitation after stroke. N Engl J Med 2005, 352: 1677-1684. [Medline] [CrossRef]

12) Woo HS, Chang KY, Park WK: The effects of mirror therapy on the hand function recovery in chronic stroke patients. J Korean Soc Occup Ther, 2011, 19: 93-103.

13) Park YJ: The effect of mirror therapy with tasks on upper extremity function and self-care in stroke patients. Department of Occupational Therapy, Graduate School, Inje University, 2012.

14) Lee YM: The effect of computer-assisted cognitive rehabilitation training and balance exercise on cognition, visual perception, hand function, balance and electroencephalography in the elderly. Department of Physical Therapy, Graduate School, Daegu University, 2011.

15) In TS, Jung KS, Lee SW, et al.: Virtual reality reflection therapy improves motor recovery and motor function in the upper extremities of people with chronic stroke. J Phys Ther Sci, 2012, 24: 339-343. [CrossRef] 